\title{
Thermospheric Studies with Mars Global Surveyor
}

\author{
F. G. Lemoine* \\ Planetary Geodynamics Laboratory, NASA GSFC, Greenbelt, Maryland USA \\ S. Bruinsma ${ }^{\dagger}$ \\ Department of Terrestrial and Planetary Geodesy, Centre Nationale D'Etudes Spatiales, Toulouse, FRANCE \\ D. S. Chinn ${ }^{\ddagger}$ \\ SGT Inc., Greenbelt, Maryland, USA \\ J. M. Forbes ${ }^{\S}$ \\ Department of Aerospace Engineering Sciences, University of Colorado, Boulder, Colorado, USA
}

The Mars Global Surveyor spacecraft has been located in a near-circular, polar, and low-altitude mapping orbit about Mars for six years, since February 1999. The spacecraft is tracked routinely by the antennae of the Deep Space Network (DSN), using the X Band radio system of the spacecraft. These tracking data have been used for routine spacecraft navigation, and for radio science studies, such as the estimation of the static and timevarying gravity field of Mars. In this paper we describe the methodology for reduction of these data in order to estimate the Mars atmospheric density (normalized to an altitude $380 \mathrm{~km}$ ) over half a solar cycle, where we discern the correlation of the density with the incident solar flux, and the 27-day solar rotation. The results show that the density at the MGS altitude varies from a mean of $0.7 \times 10^{-17} \mathrm{grams} / \mathrm{cm}^{3}$ near aphelion to a mean of 3.0 $\times 10^{-17}$ grams $/ \mathrm{cm}^{3}$ near perihelion.

\section{Nomenclature}

\author{
Subscripts \\ $i \quad$ macromodel plate index \\ $l \quad$ spherical harmonic degree \\ $m \quad$ spherical harmonic order \\ Conventions \\ $\bar{C}_{l m}, \bar{S}_{l m}$ Normalized spherical harmonic coefficients \\ $\bar{P}_{l m} \quad$ Normalized associated Legendre function \\ $\delta \bar{C}_{l m}, \delta \bar{S}_{l m}$ Variations in the spherical harmonic coefficients, $\bar{C}_{l m}$ and $\bar{S}_{l m}$ \\ $\Delta V \quad$ Velocity impulse \\ $\hat{n} \quad$ Plate surface unit normal vector \\ $\hat{s} \quad$ Unit vector to the radiation source \\ $\vec{v} \quad$ Satellite velocity vector relative to the atmosphere \\ $A_{i} \quad$ Area of macromodel plate $i$
}

*Research Scientist, Planetary Geodynamics Laboratory, Code 698, NASA Goddard Space Flight Center, Greenbelt, Maryland, 20771 USA, Member.

†Research Scientist, Department of Terrestrial and Planetary Geodesy, Centre National D'Etudes Spatiales, 31401 Toulouse Cedex 04, FRANCE.

¥Principal Programmer/Analyst, SGT Inc., 7701 Greenbelt Road, Suite 400, Greenbelt, Maryland, 20770, USA.

\$Professor, Department of Aerospace Engineering Sciences, UCB 429, University of Colorado, Boulder, Boulder, Colorado, 80309-0429, USA, Senior Member.

This material is declared a work of the U.S. Government and is not subject to copyright protection in the United States.2006 


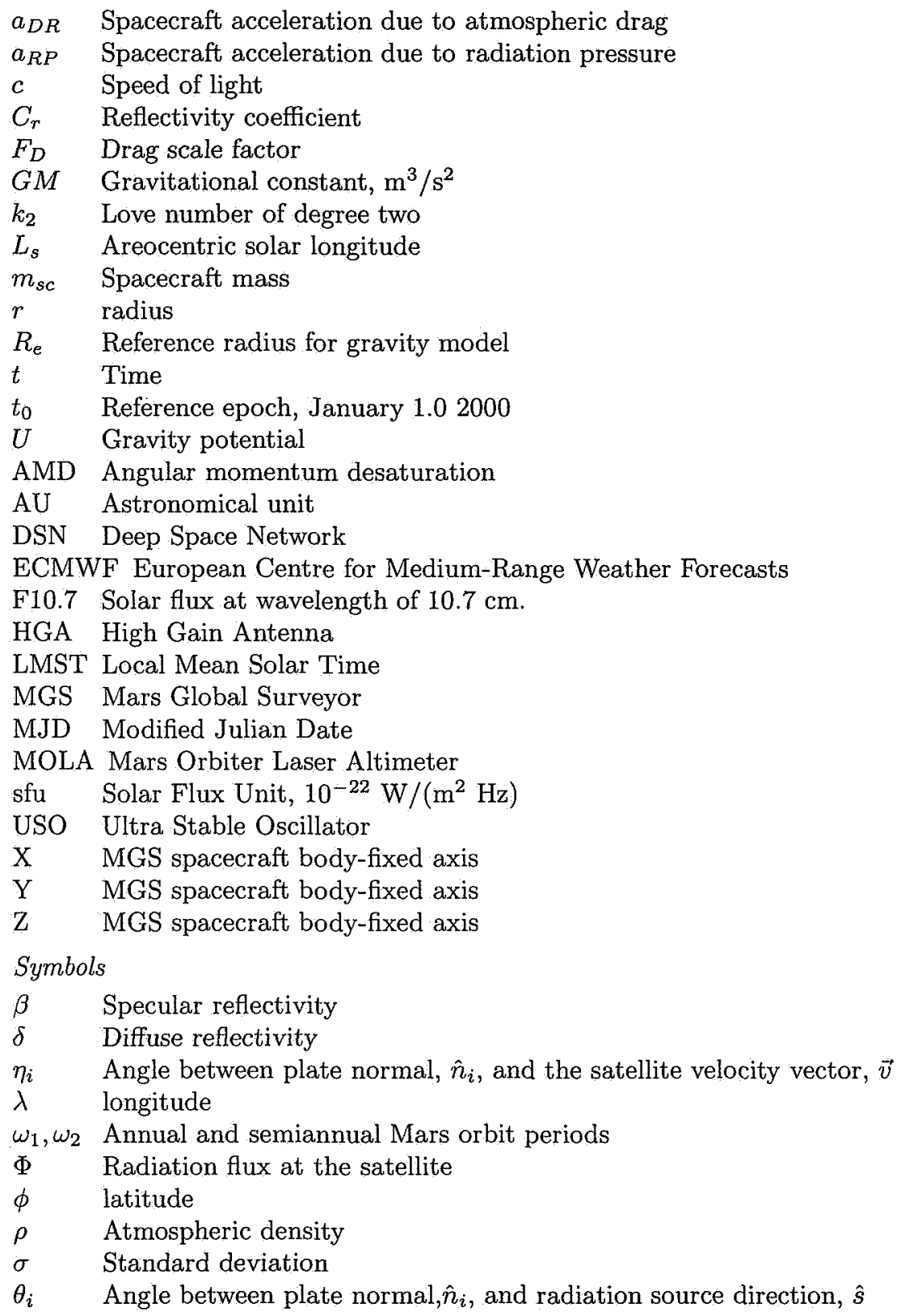

\section{Introduction}

The Mars Global Surveyor spacecraft, the successor to the Mars Observer spacecraft, arrived at Mars in September 1997. It heralded the beginning of a new age of Mars exploration, following a hiatus of nearly 16 years, since the end of the Viking Mission, and nine years since the Soviet Phobos II mission to Mars. Mars Global Surveyor had two attributes of prime importance for Radio Science: (1) the spacecraft carried an X Band communication system, compared to the S Band tracking system of the Viking Orbiters and Mariner 9 ; and (2) the spacecraft was located in a near-circular, and polar orbit at a mean altitude of $400 \mathrm{~km}$. The higher frequency of the X Band system (near $8 \mathrm{Ghz}$ ) permitted the routine collection of tracking data with a. noise of $0.10 \mathrm{~mm} / \mathrm{s}$ (or better), compared to the noisier S Band systems (near $2 \mathrm{Ghz}$ ) available in the late 1970 's. ${ }^{1}$ In addition, the near-circular orbit permitted a near-uniform and routine synoptic mapping of the planet that was not possible from the Vikings and Mariner 9 since those spacecraft were located in highly elliptical near-12-hr and near-24-hr orbits.

The highly precise data from MGS, and Mars Odyssey, have permitted the development of refined models

$$
2 \text { of } 13
$$


of the Mars gravity field. ${ }^{2-5}$ Solutions have also been developed for the time variations in the gravity field of $\mathrm{Mars}^{5-7}$ and to refine the ephemeris of Mars, ${ }^{5,8}$ and to estimate the Mars $k_{2}$ Love number. ${ }^{5,7,9}$

In the reduction of tracking data, it is necessary to model all the forces acting on the orbiting spacecraft. Atmospheric drag is modelled using the modified Stewart atmosphere model ${ }^{10}$ in the case of GMM2B and MGM1041c gravity solutions, ${ }^{2,4}$ and a version of the MARSGRAM model ${ }^{11}$ in the case of the JPL solutions. ${ }^{5}$ The solved-for drag scale coefficients describe corrections to the underlying atmospheric density model used in the precision orbit determination. In this paper we review the derivation of the Mars atmospheric density from analysis of MGS data for six years (3 Mars years) since the entry of the spacecraft into the low altitude mapping orbit in February 1999.

\section{Data and Methodology}

\section{II.A. Data Description}

The primary data for Radio Science are the tracking data of Mars Global Surveyor obtained by the Deep Space Network (DSN). The observables include Doppler and Range data from the three Deep Space Complexes at Canberra (Australia), Madrid (Spain) and Goldstone (California). ${ }^{1}$ The Doppler data include both 1-way, 2-way, and 3-way Doppler. The 2-way Doppler data involve the same DSN station for transmission and reception. The 3way Doppler data involve different DSN stations for transmission and reception. The 1-way Doppler data originate on the MGS Ultra Stable Oscillator (USO), whose primary purpose is to support the MGS radio occultation experiment. ${ }^{1}$ No 1-way data

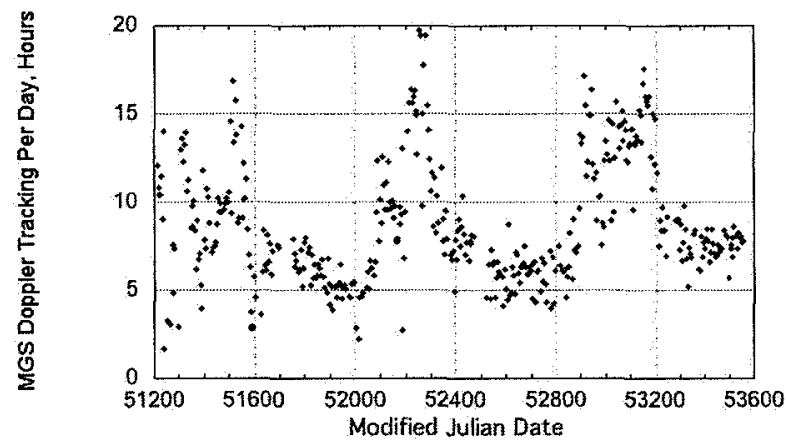

Figure 1. Hours of daily Doppler tracking for Mars Global Surveyor. for orbit determination purposes were collected prior to March 2000, however afterwards, these data constitute on average 45 percent of the data in a given data processing arc. We processed the tracking data in arcs (segments) with an average length of 4.65 days. The arclengths were meticulously chosen based on the distribution of the 2-way tracking data, with every arc always starting and stopping with a pass of 2-way Doppler data. In addition, a further constraint was to select start and stop times so as to avoid AMD events close to the arc endpoints which would be poorly determined or aliased with the initial state vector of the arc.

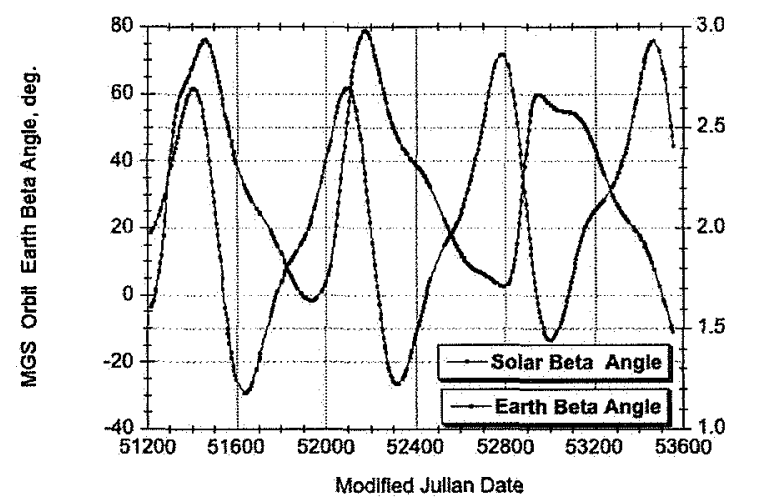

Figure 2. Earth beta angle and solar beta angle for Mars Global Surveyor from 1999 to 2005. An Earth beta angle of $0^{\circ}$ corresponds to edge-on orbit geometry, whereas an Earth beta angle of $90^{\circ}$ corresponds to face-on orbit geometry. of sight, and Earth beta angle of $0^{\circ}$ ) to nearly face-on (Earth beta angle of $90^{\circ}$ at different phases of the mission. The orbit orientation determines the sensitivity of the Doppler data and the recovery of geophysical and orbit parameters. For example, the Mars gravitational constant, $G M$, is best determined during an

We included data from the Gravity Calibration Orbit (February 1999), the period of fixed high gain antenna mapping (March 1999), and regular mapping mission (after April 1999) through July 7, 2005. A total of 401 data arcs were selected for this study. We avoided data in the vicinity of solar conjunction (July 2000, August 2002, and September 2004) since the high noise due to the solar plasma caused the quality of the orbit solutions to deteriorate. We also did not use tracking data taken while the spacecraft was in contingency mode or safe mode. The quantity of DSN data ranged from near continuous tracking during some mission phases (except for occultations by Mars as seen from Earth) to as little as 5 hrs of contact time per day (See Fig. 1). The orbit geometry experienced by MGS is depicted in Fig. 2. The orientation of the MGS orbit Earth beta angle varied from edge-on (orbit plane parallel to the line

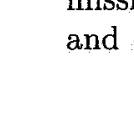


edge-on orbit orientation. ${ }^{2}$ MGS is located in a sun-synchronous orbit at approximately 2 PM. However, as illustrated in Fig. 2, the actual LMST varies by $\pm 40-50$ minutes every Mars year due to the eccentricity of the Mars orbit.

We processed the MGS tracking data using the NASA GSFC GEODYN orbit and geodetic parameter estimation program, which is a batch least-squares orbit determination filter that can process both terrestrial and interplanetary tracking data. ${ }^{12}$

\section{II.B. Force Modelling}

The reduction of the tracking data requires that we model all the forces that act on the spacecraft orbit. We model the Mars gravity field to $90 \times 90$ in spherical harmonics using MGM1041c, a gravity model based on MGS data through May $2002 .{ }^{4}$ We model the Mars gravity field with normalized coefficients $\left(\bar{C}_{l m}, \bar{S}_{l m}\right)$ using the equation ${ }^{13}$

$$
U=\frac{G M}{r}+\frac{G M}{r} \sum_{n=2}^{\infty} \sum_{m=0}^{l}\left(\frac{R e}{r}\right)^{l} \bar{P}_{l m}(\sin \phi)\left[\bar{C}_{l m} \cos (m \lambda)+\bar{S}_{l m} \sin (m \lambda)\right]
$$

where $G M$ is the universal constant of gravitation times the mass of Mars, $l$ is the degree, $m$ is the order, $\vec{P}_{l m}$ are the fully normalized associated Legendre polynomials, $R_{e}$ is the reference radius of Mars $(3397 \mathrm{~km}$ for MGM1041c), $\phi$ is the latitude, and $\lambda$ is the longitude. By definition, the degree one terms are zero, since we choose the origin of the coordinate system to be at the center of mass of the planet.

Other forces modelled include the third body perturbations due to the Sun, planets, and satellites of Mars, Phobos and Deimos, and the Mars solid tide using an a priori $k_{2}$ Love number of 0.10 .

MGS uses momentum wheels to maintain its nominal attitude. Since thruster firings from momentum wheel desaturations can perturb the orbit, we adjust accelerations radial, along-track, and cross-track to the orbit at the times of the AMD maneuvers. Early in the mapping mission, these maneuvers occurred as frequently as 3 to 4 times per day. However, after August 16, 2001, the spacecraft was no longer maintained in a strictly nadir orientation ( $+\mathrm{Z}$ axis with the instrument deck facing nadir). Rather, the $\mathrm{Z}$-axis was pitched $16^{\circ}$ from nadir in the orbit plane. This 'Relay-16' configuration had the effect of reducing the frequency of the AMD desaturations to typically one per day. ${ }^{14}$

The solar radiation pressure, planetary radiation pressure (due to the planet's albedo and thermal emission), and the atmospheric drag are modelled using a macromodel. ${ }^{15}$ The spacecraft is modelled as a series of elemental flat plates, and the contributions of each plate are vectorially summed to obtain the total acceleration. For the solar or planetary radiation pressure, the total acceleration is given by

$$
a_{R P}=-\frac{\Phi}{c} C_{r} \sum_{i=1}^{i_{\max }} \frac{A_{i} \cos \theta_{i}}{m_{s c}}\left[\left[\frac{2}{3} \delta_{i}+2 \beta_{i} \cos \theta_{i}\right] \hat{n}_{i}+\left(1-\beta_{i}\right) \hat{s}\right]
$$

where $\Phi$ is the radiation flux at the satellite, $c$ is the speed of light, $A_{i}$ is the area of the $i$ th surface plate, $\hat{n}_{i}$ is the surface normal unit vector, $\hat{s}$ is the unit vector along the direction from the satellite to the radiation source (either the Sun or a surface element of the planet), $\theta_{i}$ is the angle between the plate normal, $\hat{n}_{i}$, and the radiation source direction, $\hat{s}, \beta_{i}$ is the specular reflectivity (percent of the total incident radiation), $\delta_{i}$ is the diffuse reflectivity (percent of incident radiation), $C_{r}$ is the reflectivity coefficient (which is estimated), and Lambert's cosine law of diffuse reflection is assumed. ${ }^{16}$

The drag acceleration is modelled as,

$$
a_{D R}=-\frac{1}{2} \times \rho \vec{v}|v| \frac{F_{D}}{m_{s c}} \sum_{i=1}^{i_{\max }} A_{i} \cos \eta_{i}
$$

where $\rho$ is the atmosphere density, $\vec{v}$ is the satellite velocity vector, $|v|$ is the satellite velocity magnitude, $F_{D}$ is the drag scale coefficient (which is estimated), $m_{s c}$ is the spacecraft mass, $A_{i}$ is the area of the $i$ th plate, and $\eta_{i}$ is the angle between the surface normal unit vector, $\hat{n}_{i}$, and the velocity vector, $\vec{v}$. The spacecraft mass is provided by the spacecraft team at Lockheed Martin, and varies from $735.9 \mathrm{~kg}$ in February 1999 to $697.1 \mathrm{~kg}$ in June 2005 due to fuel consumption from orbit trim maneuvers and AMD events.

We derive the a priori atmospheric densities from the modified Stewart atmosphere model. ${ }^{10}$ In the original Stewart model, the F10.7 solar flux was modelled as an analytic function. Instead, we use the

$$
4 \text { of } 13
$$


actual F10.7 flux smoothed over three solar rotations (81 days) to drive the a priori atmospheric density model. The planetary radiation pressure uses spherical harmonic models defined over different areocentric solar longitudes, $L_{s}$, to account for the seasonal variations. ${ }^{17}$ We use a 12-plate macromodel for MGS: six for the spacecraft bus, four for the Y+ and Y-solar arrays (front and back), and two for the high gain antenna (front and back). We obtained a priori values from Lockheed Martin, and tuned these using 18 months of MGS data. ${ }^{18}$ In this paper, we describe the results of a new tuning for some of the macromodel parameters. The use of a macromodel requires detailed knowledge of the spacecraft attitude and how the articulating appendages (the $\mathrm{Y}+$ and $\mathrm{Y}$ - solar arrays and the high gain antenna) are oriented with respect to the spacecraft and in inertial space. We use spacecraft attitude data in the form of quaternions supplied to the MGS project, and we fill in the quaternions during telemetry data gaps.

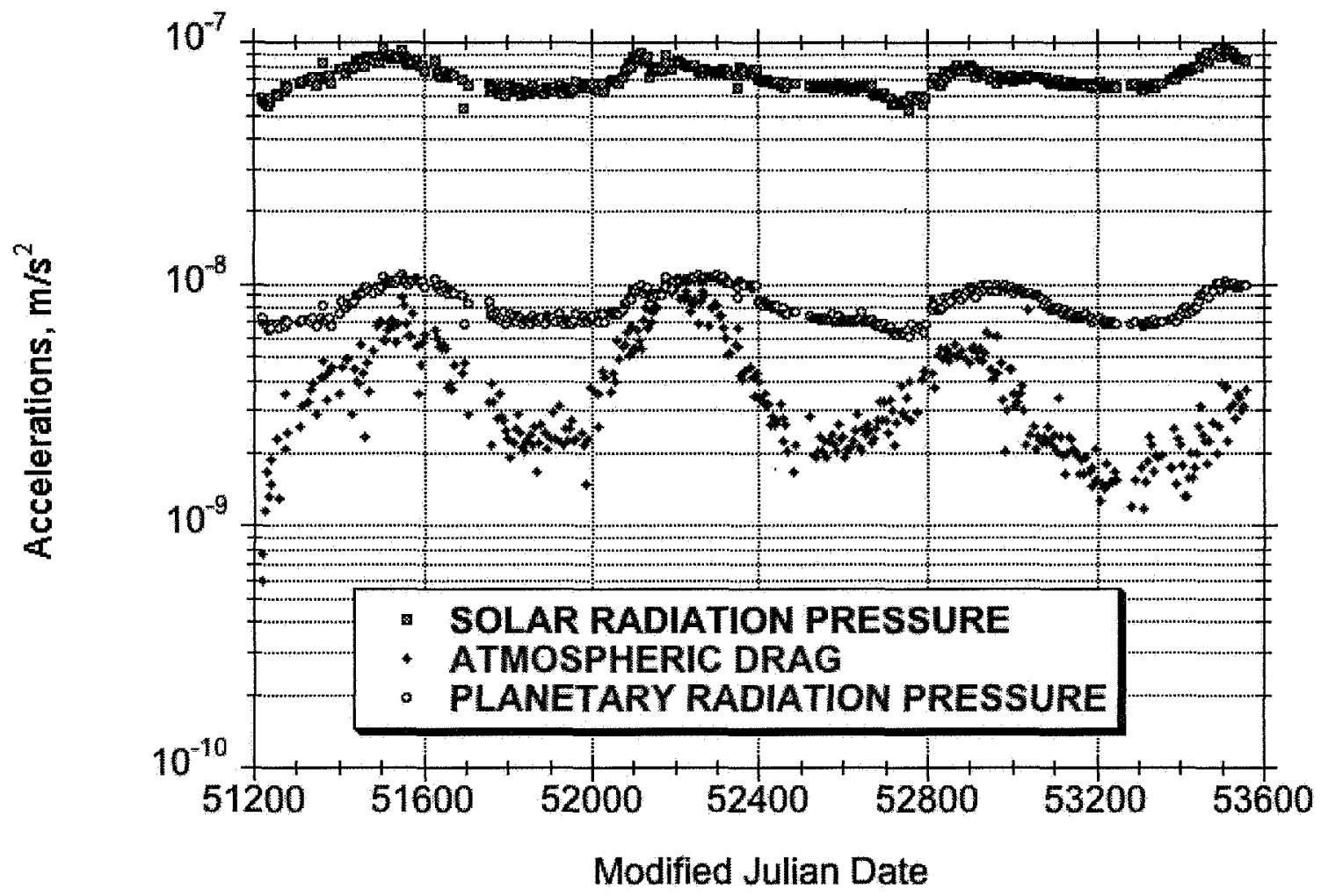

Figure 3. Nonconservative accelerations on MGS, 1999 to 2005

In Fig. 3 we depict the nongravitational accelerations calculated from daily averages for MGS from 1999 through 2005. The mean accelerations are $8.4 \times 10^{-9} \mathrm{~m} / \mathrm{s}^{2}$ for the planetary radiation pressure, $7.1 \times 10^{-8}$ $\mathrm{m} / \mathrm{s}^{2}$ for the solar radiation pressure, and $3.8 \times 10^{-9} \mathrm{~m} / \mathrm{s}^{2}$ for atmospheric drag. Due to seasonal variations and the change in the solar activity over the solar cycle, the atmospheric drag acceleration on MGS varies between $6.0 \times 10^{-10} \mathrm{~m} / \mathrm{s}^{2}$ and $1.0 \times 10^{-8} \mathrm{~m} / \mathrm{s}^{2}$, or a factor of 3.3 . The radiation pressure perturbations vary by a factor of 1.8 between Mars perihelion and aphelion. The amplitude of the accelerations might imply that the radiation pressure perturbation will swamp the perturbation due to atmospheric drag. This is not the case, as the atmospheric drag acts primarily along-track. In contrast, the planetary radiation pressure acts primarily in the radial direction, and for the MGS orbital geometry (sun-synchronous at approximately 2 PM local mean solar time), the solar radiation pressure acts primarily cross-track and radial to the orbit. The mean accelerations for two orbital arcs, one near perihelion, and one near aphelion are summarized in Table 1. The ability to estimate a drag scale factor, and hence the density variations will depend on the length of period chosen for the estimate of mean density, and the quality of the orbit solutions that are obtained. 
Table 1. Mean Radiation Pressure and Drag Accelerations on MGS for Test Arcs

\begin{tabular}{ccccccc}
\hline & \multicolumn{3}{c}{ Perihelion, MJD 51517-51522, } & \multicolumn{3}{c}{ Aphelion, MJD 52551-52556, } \\
\hline & Along-track & Cross-track & Radial & Along-track & Cross-track & Radial \\
\hline Solar radiation pressure* & 0.8 & -29.2 & -21.8 & 0.8 & -21.8 & -15.8 \\
Planetary radiation pressure* $^{*}$ & -0.4 & 1.8 & 10.5 & 0.1 & 0.9 & 7.0 \\
Atmospheric drag* & -6.9 & 0.1 & 0.01 & -2.3 & -0.003 & -0.0007 \\
\hline
\end{tabular}

*Accelerations are in units of nanometers $/ \mathrm{s}^{2}$.

\section{II.C. Measurement Modelling}

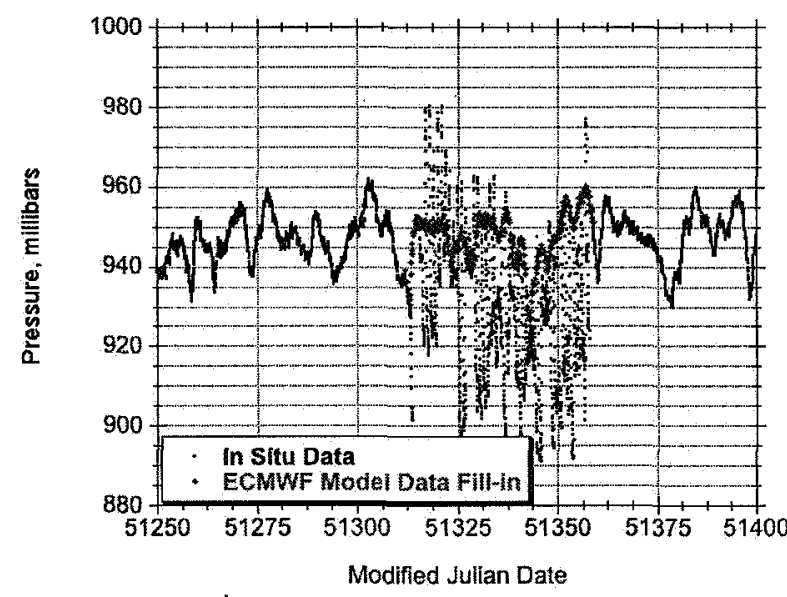

Figure 4. Actual and ECMWF weather model pressure data for the Canberra DSN complex (Tidbinbilla) in 1999. The ECMWF data were used to replace spurious in situ measurements.

for Jupiter, and 0.1 to $0.5 \mathrm{~m}$ for Saturn 17 In addition between coordinate and atomic time. ${ }^{19}$

We use the Mars IAU2000 reference system. ${ }^{20}$ A new Mars reference system has been proposed that uses a different sequence of rotation angles and whose parameters are derived from Viking Lander, Pathfinder and other data. ${ }^{5}$ Work is underway to implement this model in our GEODYN orbit determination program, however it was not available to support this present analysis of MGS data.

\section{II.D. Initial Orbit Determination Results}

The adjusted parameters in a data arc included the spacecraft state, accelerations to model the AMD events, a solar radiation reflectivity coefficient $\left(C_{r}\right)$, daily drag scale coefficients $\left(F_{D}\right)$ in most arcs, pass-bypass frequency biases for the 1-way and 3-way data, and pass-by-pass biases for the range data. The average RMS of fit was $0.216 \mathrm{~mm} / \mathrm{s}$ for the 2-way and 3-way data (computed over 401 arcs), and $0.204 \mathrm{~mm} / \mathrm{s}$ for the 1-way data (computed over 326 arcs). Arcs close to opposition had the lowest RMS of fit, 0.14 to 0.18 $\mathrm{mm} / \mathrm{s}$. Even though we did not process arcs within several weeks of solar conjunction, the increased noise is still evident in the data (see Fig. 5). The mean reflectivity coefficient, $C_{r}$ was 1.041 with a $\sigma$ of 0.093 , but exhibited distinct Mars seasonal variations with a minimum of 0.78 and a maximum of 1.26. From Table 1 , we see that near aphelion, the mean solar radiation pressure acceleration per arc can amount to 30 percent of the mean drag acceleration. Thus, if the $C_{r}$ variations are caused by orbit mismodelling, then the orbit errors could alias into estimates for the drag scale factors, and hence the estimates of atmospheric density. In order to mitigate this potential source of error, we used the MGS data to estimate macromodel reflectivity parameters, the Mars $k_{2}$ Love number, as well as annual and semiannual variations in the leading zonal 


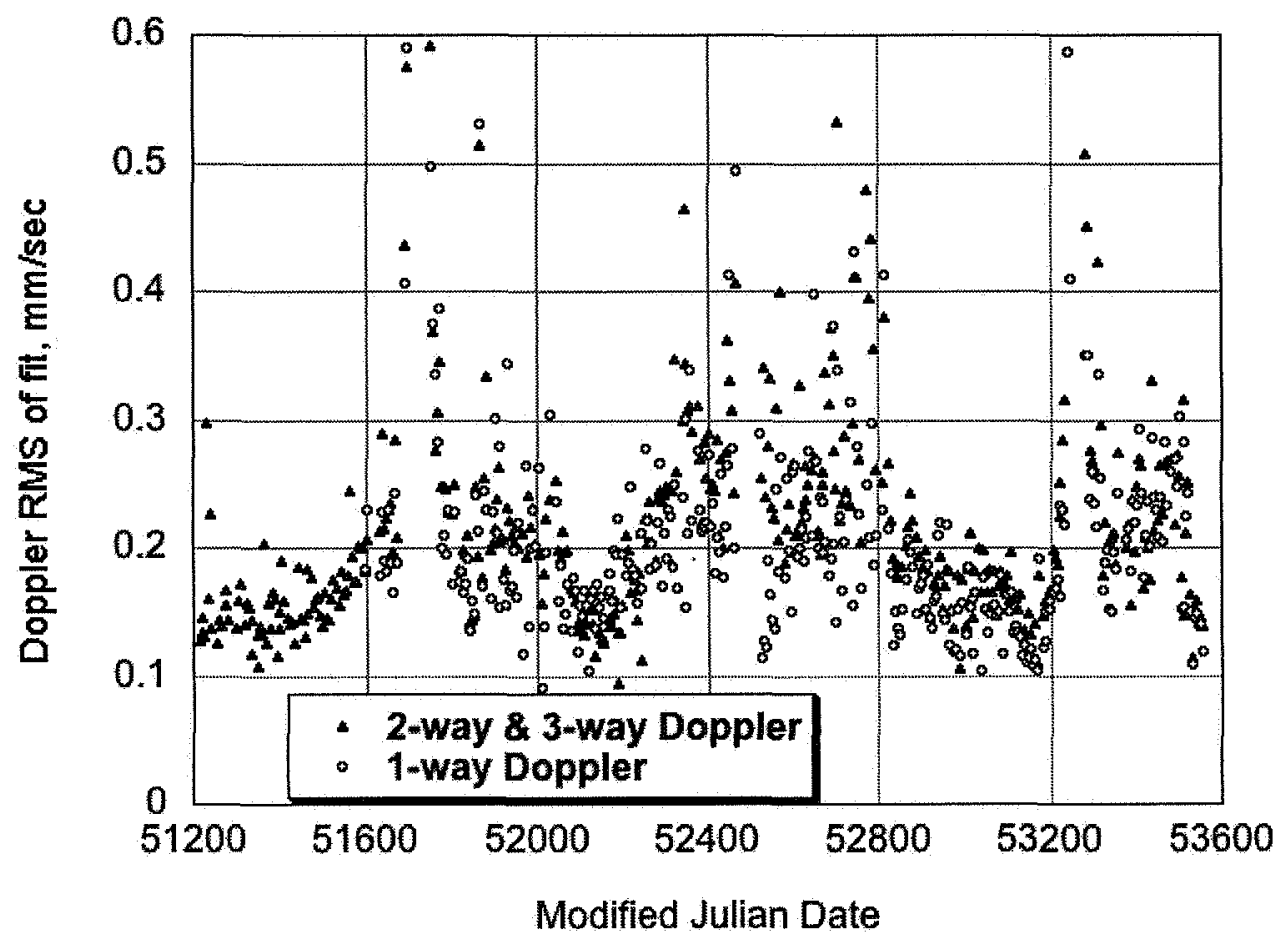

Figure 5. Doppler RMS of fit for MGS arcs from February 1999 through June 2005.

harmonics, $\bar{C}_{20}$ and $\bar{C}_{30}$. We then recomputed the MGS data arcs with these updated parameters. These parameter estimations are described in the next section.

\section{II.E. Estimation of Geophysical and Macromodel Parameters}

A major attribute of the Mars atmosphere is the annual condensation and sublimation of a sizable fraction of the atmospheric mass. This process produces variations in the Mars gravity field which can be sensed by orbiting spacecraft such as MGS and Mars Odyssey. ${ }^{5-7}$ The most significant of these gravity field variations are in the zonal harmonics. We choose to model these zonal variations according to the equations

$$
\delta \bar{C}_{20}=A_{1} \cos \omega_{1}\left(t-t_{0}\right)+B_{1} \sin \omega_{1}\left(t-t_{0}\right)+A_{2} \cos \omega_{2}\left(t-t_{0}\right)+B_{2} \sin \omega_{2}\left(t-t_{0}\right)
$$

and

$$
\delta \bar{C}_{30}=D_{1} \cos \omega_{1}\left(t-t_{0}\right)+E_{1} \sin \omega_{1}\left(t-t_{0}\right)+D_{2} \cos \omega_{2}\left(t-t_{0}\right)+E_{2} \sin \omega_{2}\left(t-t_{0}\right)
$$

where $\delta \bar{C}_{20}$ and $\delta \bar{C}_{30}$ are the variations in the normalized zonal harmonics, $\omega_{1}$ and $\omega_{2}$ are the annual and semiannual Mars periods, $A_{1}, B_{1}, A_{2}$ and $B_{2}$ are the cosine and sine coefficient components of the annual and semiannual variation for $\delta \bar{C}_{20}$. Similarly, $D_{1}, E_{1}, D_{2}, E_{2}$ are the cosine and sine coefficient components of the annual and semiannual variations for $\delta \bar{C}_{30}$. The reference epoch $t_{0}$ is January 1.0, 2000, and the argument $t-t_{0}$ is expressed in days since the reference epoch. We express the variations with respect to the mean values for $\bar{C}_{20}$ and $\bar{C}_{30}$ from the MGM1041c gravity model. ${ }^{4}$

We estimated numerous combinations of macromodel and geophysical parameters. For the macromodel, we only chose those parameters to which the MGS data seemed most sensitive, and we rejected those solutions where the macromodel reflectivity estimates were unrealistic. In addition to the periodic zonal terms, $\delta \bar{C}_{20}$ and $\delta \bar{C}_{30}$, the Mars $k_{2}$ Love number, we also adjusted the GM's of Mars and Phobos, although the GM of Phobos changed negligibly from the a priori value of $720,000 \mathrm{~m}^{3} / \mathrm{s}^{2}$. The MGS data did not have any sensitivity to the Deimos $G M$. We tested solutions for the annual and semiannual variations in $\delta \bar{C}_{21}$ and $\delta \bar{S}_{21}$, but found those to be at the edge of significance. The final geophysical parameter estimates are listed 
in Table 2. In Table 2, we also list the formal solution standard deviation scaled by a factor of ten, and the percent variation in each parameter estimate from the different trial solutions. The MGS data determine most robustly the $\delta \bar{C}_{30}$ variations followed by the $\delta \bar{C}_{20}$ semiannual variation. As long as we use the entire set of data ( 3 Mars years), and we vary only the sets of parameters estimated, with the exception of the $\delta \bar{C}_{20}$ annual terms, the geophysical parameter estimates appear quite stable. If the solutions are attempted using two 3-year (approximately 1.4 Mars years) subsets (April 1999 to June 2002; July 2002 to June 2005), the recovered values show little consistency with the solutions using the full six years of data. The full six year data set is necessary to resolve the estimated parameters, since they all (some by definition) have annual or semiannual signals, especially in a sun-synchronous orbit. We note that our recovery of Mars $k_{2}$ is quite similar to that of Ref. 5. The extremely poor determination of the $\delta \bar{C}_{20}$ annual variation is likely due to the deleterious effect of the AMD's which contaminate the parameter recovery.

Table 2. Estimated Geophysical Parameters from MGS Data, 1999-2005

\begin{tabular}{lcccc}
\hline Parameter & A priori value & Estimate & Error & Percent variation \\
\hline$A_{1}, \delta C_{20}$ annual cosine & 0 & $6.218 \times 10^{-11}$ & $1.3 \times 10^{-9}$ & 224 \\
$B_{1}, \delta C_{20}$ annual sine & 0 & $-4.317 \times 10^{-10}$ & $1.5 \times 10^{-9}$ & 23 \\
$A_{2}, \delta C_{20}$ semiannual cosine & 0 & $1.531 \times 10^{-9}$ & $1.4 \times 10^{-9}$ & 9.6 \\
$B_{2}, \delta C_{20}$ semiannual sine & 0 & $1.105 \times 10^{-9}$ & $1.4 \times 10^{-9}$ & 14.1 \\
$D_{1}, \delta C_{30}$ annual cosine & 0 & $2.233 \times 10^{-9}$ & $8.5 \times 10^{-11}$ & 0.5 \\
$E_{1}, \delta C_{30}$ annual sine & 0 & $1.946 \times 10^{-9}$ & $7.1 \times 10^{-11}$ & 0.9 \\
$D_{2}, \delta C_{30}$ semiannual cosine & 0 & $4.088 \times 10^{-10}$ & $7.5 \times 10^{-11}$ & 1.2 \\
$E_{2}, \delta C_{30}$ semiannual sine & 0 & $3.111 \times 10^{-10}$ & $7.7 \times 10^{-11}$ & 5.5 \\
$k_{2}$ & 0.10 & 0.176 & 0.041 & 3.9 \\
Mars $G M\left(\mathrm{~m}^{3} / \mathrm{s}^{2}\right)$ & $4.282837024 \times 10^{13}$ & $4.282836798 \times 10^{13}$ & $1.37 \times 10^{6}$ & $1.4 \times 10^{-6}$ \\
\hline
\end{tabular}

* We report the formal errors of the least squares solution scaled by a factor of ten.

t The percent variation is derived from examination of the variation in the parameter estimates from multiple solutions.

Table 3. MGS Macromodel Adjuștments

\begin{tabular}{lccl}
\hline Plate & $\begin{array}{c}\text { Area } \\
\left(\mathrm{m}^{2}\right)\end{array}$ & $\begin{array}{c}\text { A priori* }^{*} \\
\text { Reflectivity } \\
(\beta, \delta)\end{array}$ & $\begin{array}{l}\text { Adjusted } \\
\text { Reflectivity } \\
(\beta, \delta)^{\ddagger}\end{array}$ \\
\hline +X Bus & 3.30 & $0.13,0.52$ & $\delta=0.3165$ \\
-X Bus & 3.30 & $0.13,0.52$ & $\delta=0.4008$ \\
+Y Bus & 3.56 & $0.13,0.52$ & $\ldots$ \\
-Y Bus & 3.56 & $0.13,0.52$ & $\ldots$ \\
+Z Bus & 2.31 & $0.13,0.52$ & $\ldots$ \\
-Z Bus & 2.31 & $0.13,0.52$ & $\ldots$ \\
Solar arrays (front) & 15.70 & $0.0494,0.1976$ & $\beta=0.0653$ \\
Solar arrays (back) & 15.70 & $0.0790,0.2822$ & $\delta=0.4494$ \\
HGA (front) & 1.94 & $0.10,0.40$ & $\cdots$ \\
HGA (back) & 1.94 & $0.13,0.52$ & $\ldots$ \\
\hline
\end{tabular}

${ }^{*}$ A priori macromodel values from Ref. 18.

$\dagger$ The $\mathrm{Y}+$ and $\mathrm{Y}$ - solar arrays are treated as a unit in the macromodel adjustment.

$\ddagger$ Only the adjusted reflectivity values are listed.

The macromodel reflectivity parameter estimates are given in Table 3 . In the MGS spacecraft coordinate system during normal mapping operations (before the start of 'Relay-16' mode), +Z refers to the instrument 
deck portion of the spacecraft bus and faces nadir, $+Y$ and $-Y$ face the cross-track directions, $+X$ and $-X$ face in the velocity and anti-velocity directions. The solar arrays extend along the $+Y$ and $-Y$ axes and normally track the Sun. The high gain antenna is located on a boom extending in the $-\mathrm{Z}$ direction, and tracks the Earth using a dual-axis gimbal. Near the subsolar point, the front sides of the solar arrays face the Sun, and the back sides of the solar array face the planet when the planetary radiation pressure perturbation is at its peak. The solar array reflectivity values are composites that represent the contribution of the inner and outer solar arrays, the drag flaps, and the yoke. ${ }^{23}$ The $Y+$ and $Y$ - solar array parameters are tied together since we cannot obtain separate estimates for each panel. From previous experience, we know that the most important parameter to adjust in order to reduce macromodel error is the specular reflectivity of the solar array. ${ }^{24,25}$ The diffuse reflectivity of the back side of the solar arrays was adjusted to accommodate errors in the planetary radiation pressure model. An analysis of the information matrix prior to inversion also indicated that the $+\mathrm{X}$ and $-\mathrm{X}$ panels had heightened sensitivity, so the diffuse reflectivities for those plates were also adjusted.

\section{II.F. Results of Updated Orbit Computations}

In Table 4, we give the results of two additional series of orbit computations for the MGS data arcs: (1) applying the new time-variable gravity estimates and the new value of the $k_{2}$ Love number, and (2) applying both the new geophysical parameter estimates and the new macromodel reflectivities. The application of the $\delta \bar{C}_{20}$ and $\delta \bar{C}_{30}$ variations and the new $k_{2}$ improve the average RMS of fit by about 10 percent or $0.02 \mathrm{~mm} / \mathrm{s}$. The subsequent application of the macromodel has minimal impact on the average level of fit to the data. However, the most notable result is the reduction in the scatter of the reflectivity coefficient, $C_{r}$, as measured by the standard deviation computed over all 401 data arcs. The standard deviation, $\sigma$ is reduced from 0.093 in the original processing, to 0.065 with the application of the new geophysical parameters, and to 0.050 with the application of both the geophysical and macromodel parameters. We show the reflectivity coefficient in Fig. 6 for the original and the new time

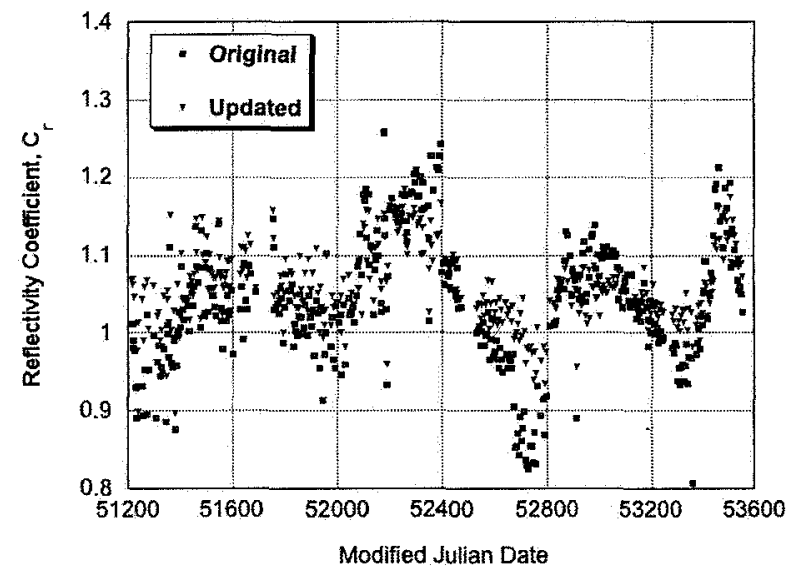

Figure 6. Radiation pressure reflectivity coefficient, $C_{r}$, for the original and the updated time series of MGS data arcs from 1999 to 2005. The updated time series includes the annual and semiannual variations in the zonal harmonics, $\delta \vec{C}_{20}$ and $\delta \widetilde{C}_{30}$, the new $k_{2}$ estimate, and the updated macromodel reflectivity parameters. series. The strong annual excursions in the reflectivity coefficient, particularly near MJD 52700, have been reduced. The average RMS difference in the orbits between the original and updated processing is $0.17 \mathrm{~m}$ in the radial direction, $4.17 \mathrm{~m}$ cross-track, and 2.63 $\mathrm{m}$ along-track. Sometimes the orbit differences between the new and updated processing can be large. For example, the cross-track orbit differences are 20-50 m whenever the Earth beta angle for the MGS orbit is less than $20^{\circ}$. This is understable as during an edge-on orbit orientation (Earth beta angle of $0^{\circ}$ ), the Doppler data have no cross-track sensitivity, and changes in the force model cross-track to the orbit will have maximum effect.

\section{Atmospheric Density Results}

We first review the drag scale factors obtained by GEODYN using the final updated orbit time series (TVG $+k_{2}+$ macromodel in Table 4). The daily drag scale factors were filtered to remove some with negative values, those whose relative errors were greater than 50 percent, or whose standard deviations were excessively large. We retained $1760 \mathrm{drag}$ scale factors out of 1794 , and show the relative error in percent in Fig. 7. The relative error is derived from the formal error of the drag scale factors in each orbit determination solution, and reflects the a priori data weighting (discussed earlier) as well as the distribution and quality of the tracking data in each data arc. It is evident that the daily drag scale factor estimates are much noisier early in the mission, when the spacecraft experienced 3-4 AMD maneuvers per day. Through August 16, 
Table 4. RMS of Fit Summary for MGS Arcs from 1999 to 2005

\begin{tabular}{|c|c|c|c|c|c|c|c|}
\hline \multirow[t]{2}{*}{ Orbit Series } & \multicolumn{2}{|c|}{$\begin{array}{c}\text { RMS, 2-way \& } \\
\text { 3-way Doppler } \\
(\mathrm{mm} / \mathrm{s})^{*}\end{array}$} & \multicolumn{2}{|c|}{$\begin{array}{c}\text { RMS, 1-way } \\
\text { Doppler } \\
(\mathrm{mm} / \mathrm{s})^{*}\end{array}$} & \multicolumn{3}{|c|}{$\begin{array}{c}\text { Reflectivity } \\
\text { Coefficient } \\
C_{r}\end{array}$} \\
\hline & Mean & Median & Mean & Median & Mean & Median & Std. Dev. ${ }^{\dagger}$ \\
\hline Original & 0.214 & 0.198 & 0.203 & 0.186 & 1.042 & 1.037 & 0.093 \\
\hline$+\mathrm{TVG}+k_{2} \ddagger$ & 0.195 & 0.182 & 0.180 & 0.165 & 1.048 & 1.040 & 0.065 \\
\hline$+\mathrm{TVG}+k_{2}+$ new macromodel ${ }^{\ddagger}$ & 0.195 & 0.182 & 0.181 & 0.164 & 1.059 & 1.054 & 0.050 \\
\hline
\end{tabular}

2001 or the period of standard mapping, the mean relative error is $6.9 \%$, the median is $5.4 \%$, and the RMS is $9.2 \%$. In contrast, during 'Relay-16' mode, the mean relative error is $2.4 \%$, the median is $1.7 \%$ and the RMS is $3.6 \%$. These relative errors are important as they will map directly into the derived densities.

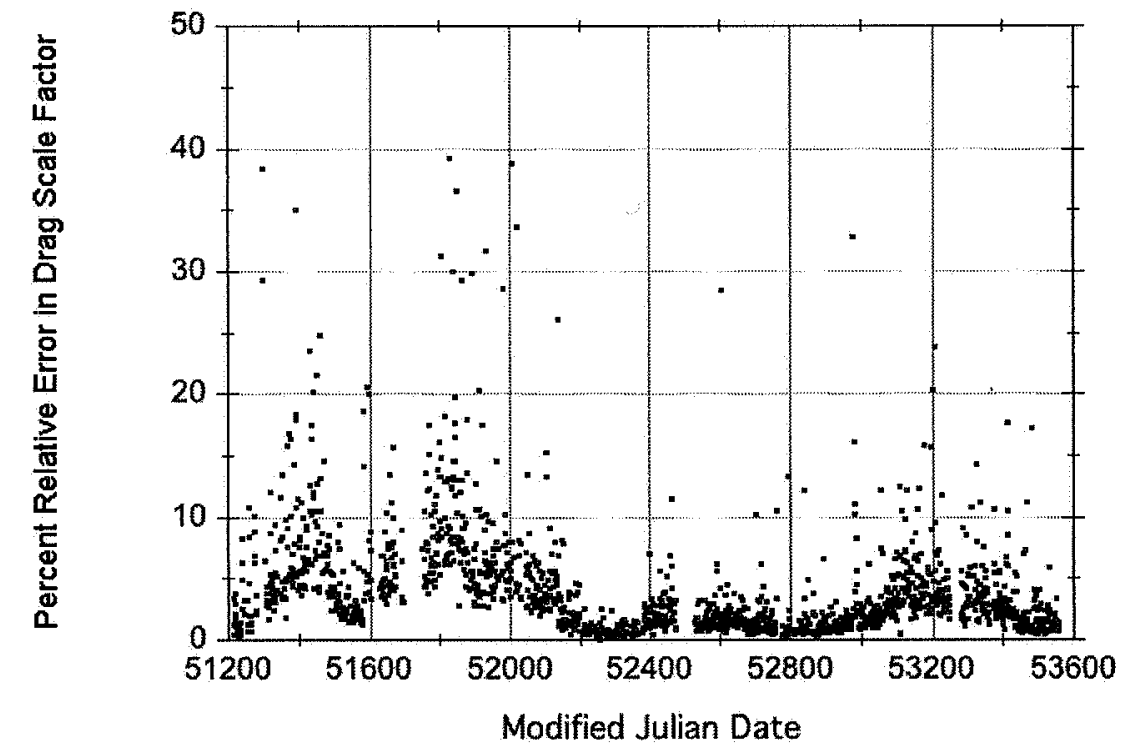

Figure 7. Percent relative error in the drag scale coefficients, $F_{D}$, estimated by GEODYN for MGS from 1999 to 2005.

The recovered densities are shown in Fig. 8 from 1999 through 2005 . We use the $10.7 \mathrm{~cm}$ radio flux as a proxy for the EUV variability that is known to drive the behaviour the planetary thermospheres. This flux, which is measured at the Earth, is corrected by scaling the daily F10.7 defined at 1 AU to the actual Mars distance from the Sun, and the variations in the Sun-Earth-Mars angle as the planets move about the Sun. ${ }^{26}$ The period from 1999 to 2005 includes the last maximum of the solar cycle 23 , and the approach to the current solar minimum. The combination of variations in solar flux, and the distance of Mars from the Sun cause the density at the MGS altitude to vary by a factor of five, from 1 to $5 \times 10^{-17} \mathrm{grams} / \mathrm{cm}^{3}$. The mean density near aphelion $\left(L_{s}=75^{\circ}\right)$ is about $0.7 \times 10^{-17}$ grams $/ \mathrm{cm}^{3}$ whereas near perihelion $\left(L_{s}=255^{\circ}\right)$ the mean density is $3.0 \times 10^{-17}$ grams $/ \mathrm{cm}^{3}$ (see Fig. 9). In addition, regional and global dust storms which generally occur within a few months of perihelion, also contribute to the density variability depicted in Fig. 8 and Fig. 9.

The density data show evidence of the Mars atmosphere responding to the changing solar flux, induced by the near 27-day solar rotation. In Fig. 10, we illustrate the behaviour of the Mars thermosphere over eight 
month time span. The recovered atmospheric density residuals clearly contain the same 27-day periodicity that is evident in the corrected F10.7 data.

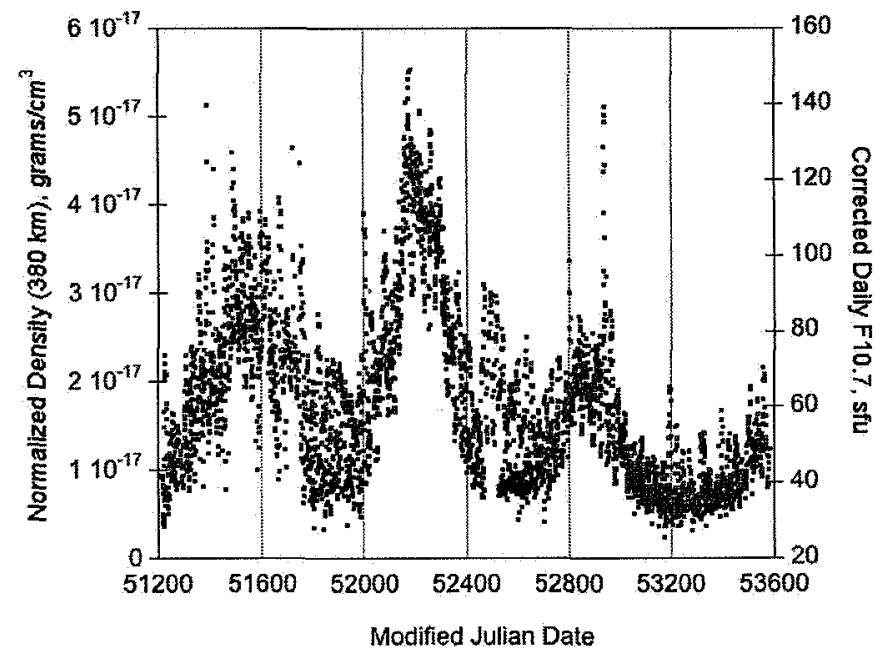

Figure 8. Normalized density at $380 \mathrm{~km}$ for MGS and corrected daily solar flux (F10.7), A solar flux unit (sfu) has units of $10^{-22} \mathrm{~W} /\left(\mathrm{m}^{2} \mathrm{~Hz}\right)$.

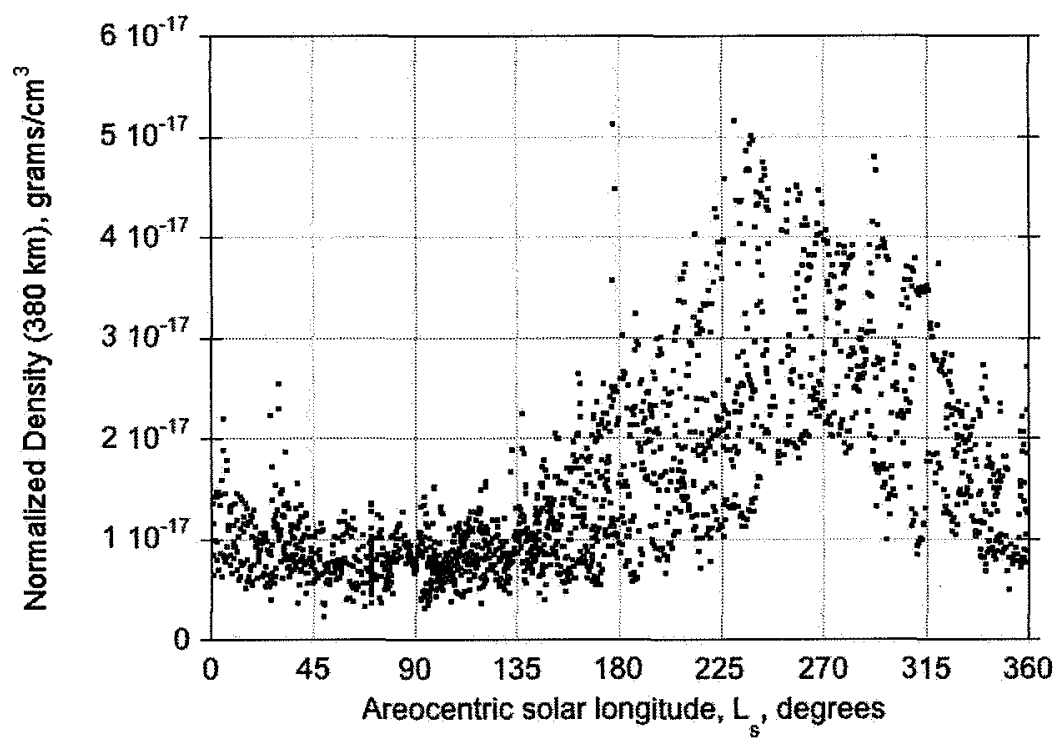

Figure 9. MGS normalized density at $380 \mathrm{~km}$ versus areocentric solar longitude, $L_{s}$.

\section{Conclusion}

We have computed the mean density at the MGS altitude over 3 Mars years, from 1999-2005. The variations in density are controlled by variations in solar flux, and modulated by both the near 27-day solar rotation, and change in solar flux over the solar cycle. Mean densities at the MGS altitude can vary by a factor of five between perihelion and aphelion.

The recovery of atmospheric density requires precise modelling of all forces acting on the MGS spacecraft. The macromodel that is used to model the variable cross-sectional area and provide for more sophisticated 


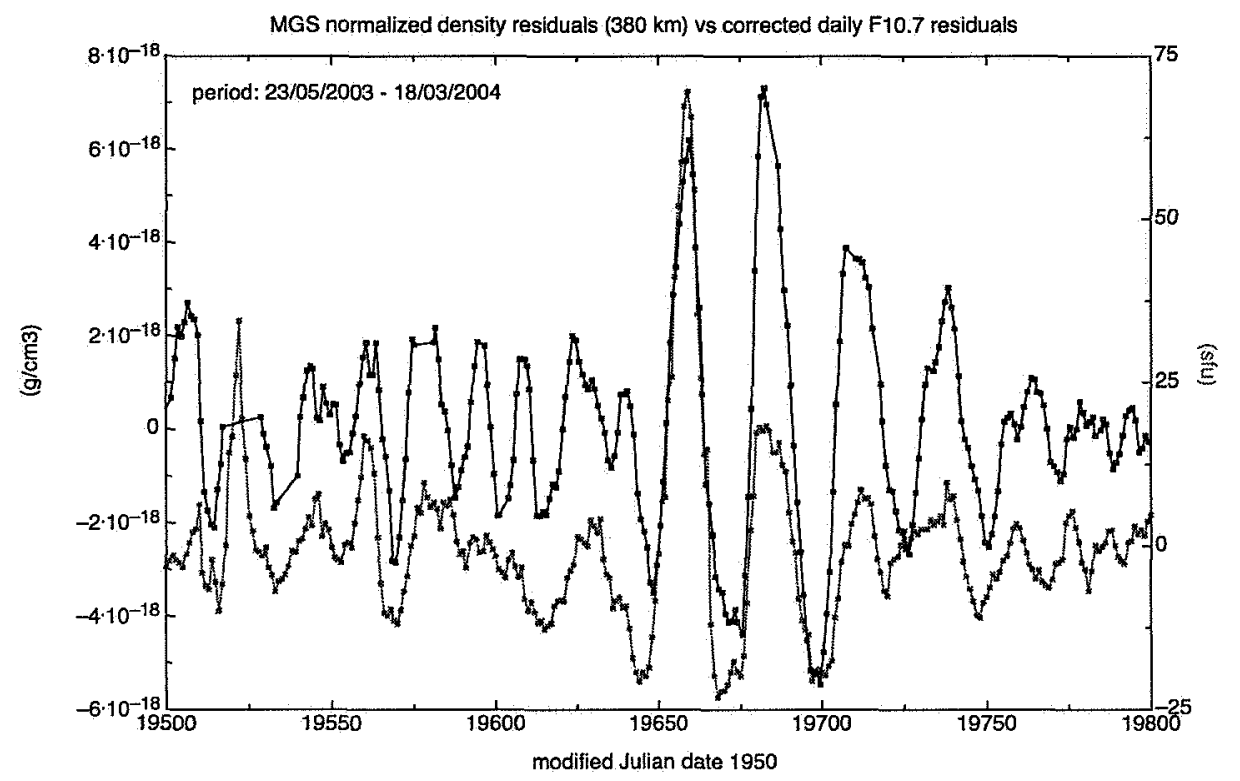

Figure 10. MGS normalized density residuals $(380 \mathrm{~km})$ vs. corrected daily F10.7 residuals for May 23,2003 to March $18,2004$.

drag and radiation pressure modelling, is a major improvement over using a simplistic model such as a cannonball. However, the macromodel does not allow for self-shadowing of spacecraft components, nor does it account for spacecraft thermal emission or the radiation interaction between different spacecraft surfaces. The a priori modelling of the zonal $\delta \bar{C}_{20}$ and $\delta \bar{C}_{30}$ variations appears to be essential in order to achieve the best possible orbits, yet in our analysis we did not obtain a reliable solution for the $\delta \bar{C}_{20}$ annual variations. If a more parsimonious strategy were followed to model the AMD's, such as using information supplied by the spacecraft team, or limiting the estimation of accelerations or $\Delta_{V}$ 's to the axes along which the AMD occurred, better solutions for the $\delta \bar{C}_{20}$ variation might be obtained. The addition of MOLA altimeter crossovers as an orbit determination data type could improve the orbit determination solutions for MGS through June 2001 and consequently would provide more robust estimates of the MGS density early in the mapping mission.

A logical extension of this analyis being pursued by the authors is to incorporate these MGS density data, along with improved solar activity and seasonal variations, and dust storm effects, into an empirical density and temperature model for the Mars thermosphere, such as DTM-Mars. ${ }^{18}$

\section{Acknowledgments}

We acknowledge the MGS Radio Science Team for making the MGS tracking data and ancillary information available. Jean-Paul Boy (University of Strasbourg, France) provided the corrected weather model data from the ECMWF. FGL acknowledges Despina Pavlis (SGT Inc.) and John McCarthy (SGT Inc.) for support of GEODYN and SOLVE.

\section{References}

${ }^{1}$ Tyler G.L., Balmino G., Hinson D.P., Sjogren W.L., Smith D.E., Simpşon R.A., Asmar S.W., Priest P, and Twicken J.D., "Radio Science Observations with Mars Global Surveyor: Orbit Insertion Through One Mars Year in Mapping Orbit," $J$. Geophys. Res., Planets, 106 (E10), 25 Oct. 2001, pp. 23327-23348.

${ }^{2}$ Lemoine F.G., Smith D.E., Rowlands D.D., Zuber M.T., Neumann G.A., Chinn D.S., and Pavlis D.E., "An Improved Solution of the Gravity Field of Mars (GMM-2B) From Mars Global Surveyor," J. Geophys. Res., Planets, 106 (E10), 25 Oct. 2001, pp. 23359-23376.

${ }^{3}$ Yuan D.N., Sjogren W.L., Konopliv A.S., and Kucinskas A.B., "Gravity Field of Mars: A 75th Degree and Order Model," J. Geophys. Res., Planets, 106 (E10), 25 Oct. 2001, pp. 23377-23401.

${ }^{4}$ Lemoine, F.G., MGM1041c Gravity Model, Mars Global Surveyor Radio Science Archival Volume MGS-M-RSS-5-SDP- 
V1/mors_1021, URL: http://pds-geosciences.wustl.edu/geodata/mgs-m-rss-5-sdp-v1/mors_1021/sha/, Geosciences Node, Planetary Data System, Washington University, St. Louis, Missouri, March 28, 2003.

${ }^{5}$ Konopliv. A.S., Yoder, C.F., Standish, E.M., Yuan, D.N., and Sjogren W.L., "A Global Solution for the Mars Static and Seasonal Gravity, Mars Orientation, Phobos and Deimos Masses, and Mars Ephemeris," Icarus, 182(1), May 2006, pp. 23-50.

${ }^{6}$ Smith, D.E. Zuber, M.T., and Neumann, G. A., "Seasonal Variations of Snow Depth on Mars," Science, 294, 2001, pp. 2141-2146.

${ }^{7}$ Balmino, G., Marty, J.C., Duron, J., and Karatekin Ö., "Mars Long Wavelength Gravity Field variations: A New Solution From MGS Tracking Data," IAG Symposia series, Vol. 130, Springer-Verlag, 2006.

${ }^{8}$ Standish, E.M., "JPL Planetary Ephemeris DE410," JPL Interoffice Memorandum, IOM 312.N-03-009, Jet Propulsion Laboratory, Pasadena, California, April 24, 2003.

${ }^{9}$ Yoder, C.F., Konopliv, A.S., Yuan, D.N., Standish, E.M., and Folkner, W.M., "Fluid Core Size of Mars From Detection of the Solar Tide," Science, 300, 2003, pp. 299-303.

${ }^{10}$ Stewart, A.I.F., "Revised Time Dependent Model of the Martian Atmosphere for Use in Orbit Lifetime and Sustenance Studies," final report, JPL PO NQ-802429, Lab. for Atmos. and Space Phys., Univ. of Colorado, Boulder, March 26, 1987.

${ }^{11}$ Justus, C.J., Johnson, D.L., and James, B.F., "A Revised Thermosphere for the Mars Global Reference Atmosphere Model (MarsGRAM version 3.4)," NASA TM-108513, 1996.

${ }^{12}$ Pavlis, D.E., Poulose, S., and J.J. McCarthy, GEODYN Operations manuals, contractor report, SGT Inc., Greenbelt, Maryland, 2006.

${ }^{13}$ Kaula, W.M., Theory of Satellite Geodesy: Applications of Satellites to Geodesy, Dover Publications, Inc., Mineola, New York, 2000.

${ }^{14}$ Esposito, P.B., Bhat, R., Demcak, S. Ardalan S., Breeden, J., Helfrich G., Jefferson, D., and Stauch, J., "Mars Global Surveyor and Mars Odyssey- Relay Satellites for the Mars Exploration Rover Mission," AIAA Paper 2004-5094, AIAA/AAS Astrodynamics Specialist Conference, Providence, Rhode Island, August 16-19, 2004.

${ }^{15}$ Marshall, J.A.M., and Luthcke, S.B., "Radiative Force Model Performance for TOPEX/Poseidon Precision Orbit Determination," J. Astron. Sci., 45(2), 1994, pp. 229-246.

${ }^{16}$ Miliani, A., Nobili A.M., and Farinella, P. Non-gravitational Perturbations and Satellite Geodesy, Adam Hilger, 1987.

${ }^{17}$ Lemoine, F.G.R., "The Dynamics of Orbiting Satellites and Gravity Model Development," Dept. of Aerospace Engineering Sciences, University of Colorado, Boulder, Colorado, 1992.

${ }^{18}$ Bruinsma, S. and Lemoine, F.G., "A Preliminary Semiempirical Thermosphere Model of Mars: DTM-Mars," J. Geophys. Res., Planets, 107(E10), 2002, doi:10.1029/2001JE001508.

${ }^{19}$ Moyer, T.D., "Transformation from Proper Time on Earth to Coordinate Time in the Solar System Barycentric SpaceTime Frame of Reference: Part I and Part II, Celestial Mech.,, 23, 1981, pp. 33-68.

${ }^{20}$ Seidelmann, P.K., Abalakin, V.K., Bursa, M., Davies, M.E., De Bergh, C., Lieske, J.H., Oberst, J., Simon, J.L., Standish, E.M., Stooke, P., and Thomas P.C., "Report of the IAU/IAG Working Group on Cartographic Coordinates and Rotational Elements of the Planets and Satellites: 2000," Cel. Mechanics and Dyn. Astron., 82(1), January 2002, pp. 83-110.

${ }^{21}$ Sniffin, R.W., "301, Rev. C, Coverage and Geometry;" DSMS Telecommunications Link Design Handbook, DSN Document 810-005, Rev. E, Jet Propulsion Laboratory, Pasadena, California, August 20, 2005.

${ }^{22}$ Hopfield, H.S., "Tropospheric Effect on Electromagnetically Measured Range: Prediction from Surface Weather Data, Radio Science, 6(3), March 1971, pp. 357-367.

${ }^{23}$ Lemoine, F.G., Rowlands, D.D., Smith, D.E., Chinn, D.S., Pavlis, D.E., Luthcke, S.B., Neumann, G.A., and Zuber, M.T., "Orbit Determination for Mars Global Surveyor During Mapping," AAS Paper 99-328, AAS/AIAA Astrodynamics Conference, Girdwood, Alaska, August 16-19, 1999.

${ }^{24}$ Marshall, J.A., Zelensky, N.P., Klosko, S.M., Chinn, D.S., Luthcke, S.B., Rachlin, K.E., and Williamson, R.G., "The Temporal and Spatial Characteristics of TOPEX/POSEIDON Radial Orbit Error," J. Geophys. Res., Oceans, 100(C12), Dec. 15,1995 , pp. 25331-25352.

${ }^{25}$ Luthcke, S.B., Marshall, J.A., Rowton, S.C., Rachlin, K.E., Cox, C.M., and Williamson R.G., "Enhanced Radiative Force Modelling of the Tracking and Data Relay Satellites," J. Astron. Sci., 45(3), Jul.-Sept. 1997, pp. 349-370.

${ }^{26}$ Forbes, J. M., Bruinsma, S., and Lemoine, F. G., "Solar Rotation Effects on the Thermospheres of Mars and Earth," Science, 312, June 2, 2006, pp. 1366-1368. 